\section{ON THE SCIENCE OF WEIGHING AND MEASURING, AND THE STANDARDS OF WEIGHT AND MEASURE*}

\section{VIII.}

$\mathrm{T}$ $\mathrm{HE}$ ordinary method of commercial weighing by putting the weights in one scale and the commodity to be weighed in the other, and then observing when a sufficient equilibrium is produced, is inadmissible for scientific weighings, as it is subject to errors arising from defects in the balance itself. To avoid any such errors, and obtain scientific precision in the results, a check is required which is found in a system of double weighing. There are two methods of double weighing for the comparison of two standard weights. One method, known as Borda's, and generally used in France, is that of substitution, or weighing first one of the standard weights to be compared, and then the other substituted for it, against a counterpoise placed in the other pan. The difference between the mean resting points of the index needle in these two weighings shows the difference of the two weights in divisions of the scale. The second method, known as Gauss's, but which was first invented by Le Père Amiot, and is now generally used in England and Germany, except for hydrostatic weighings, is that of alternation, or first weighing the two standards against each other, and then repeating the weighings, after interchanging the weights in the pans. By this second method no counterpoise weight is required, and half the difference between the mean resting points of the index needle shows the difference of the two weights, in divisions of the scale.

In all scientific weighings of standards with balances of precision, it is necessary that the weights to be compared should be so nearly equal that neither pan shall absolutely weigh down the other. The balance must merely oscillate so that the pointer does not exceed the limits of the index scale. In order to obtain an equipoise within this limit, it is requisite to provide small balance weights, most accurately verified, to be added to either pan, as may be found necessary.

The mode of reading adopted by the best authorities in the process of weighing by Gauss's method is as follows: - The comparing standard being in the left-hand pan, and the compared standard in the right-hand pan, and sufficient equipoise being obtained by adding small balance weights, if requisite, the balance is put in action, and the movement of the needle observed through a telescope. The reading at the first turn of the pointer is disregarded. The three next turns are noted, and the reading at the third turn of the pointer, and half the sum of the readings at the second and fourth turns are taken as the highest and lowest readings. Their mean is the resting point of the balance, or the reading of its position of equilibrium. The balance is then stopped, and the weights interchanged, when similar readings are taken and dealt with in the same manner. These two observations constitute one comparison. In cases where great accuracy is required, several successive comparisons are taken, in order to obtain a mean result. Some additional weighings are taken after adding a small balance weight to either pan, in order to ascertain the value of a division of the index scale. And if this balance-weight be added successively to each pan the weighings may be used as additional comparisons.

In using Gauss's method of weighing, it is very desirable to be able to transfer the pans and the weights contained in them from one end of the beam to the other without opening the balance case, and thus to avoid sudden changes of temperature of air within the balance case and consequent production of currents of air. For this pur- pose, the following plan is adopted. A grooved brass rod is fixed inside the balance case over and a little behind the beam. Upon this rod a brass slider is made to traverse by being attached to a slender brass rod drawn backwards or forwards from the outside of the case. A descending wire with a hook at the end is attached to the slider. For changing the weights, the slider and hook are brought to the right-hand end of the beam, when the pan and weight are lifted from the beam and transferred to the hook by means of a brass rod curved at the end and introduced through a small hole at the side of the balance case. The pan and weight are then slid to the other end of the beam, when the left-hand pan and weight are lifted in a similar manner from the beam and the right-hand pan and weight substituted. It only remains then to transfer the left-hand pan and weight to the right-hand end of the beam.

This method possesses a further advantage. In making a great number of comparisons between two standard weights, they are exposed to some risk of being injured

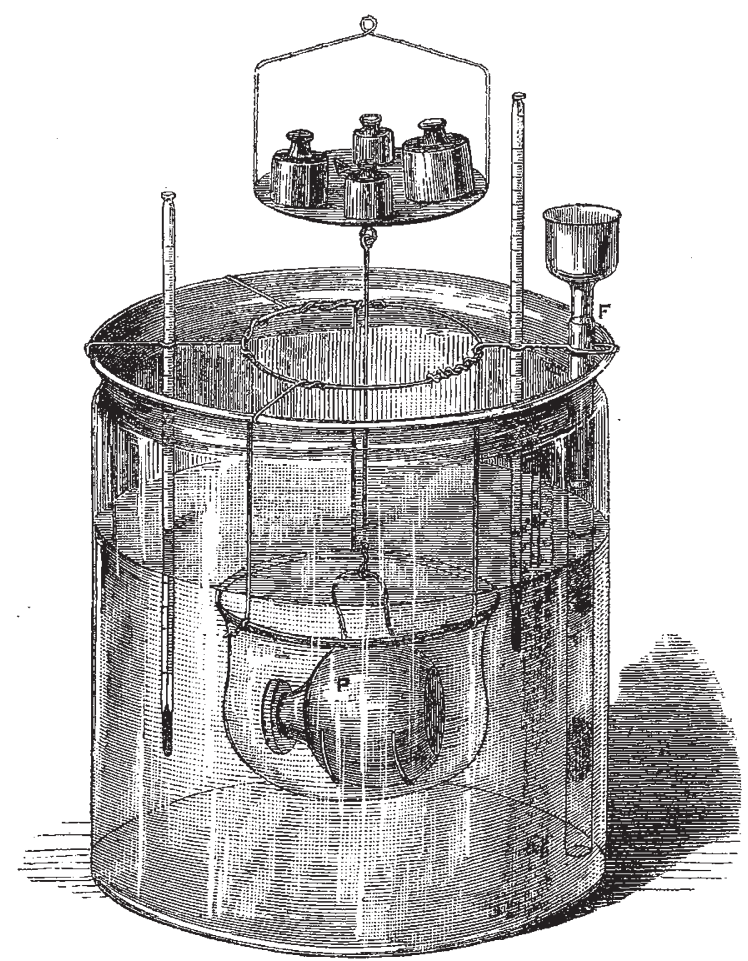

FIG. 18.-Mode of hydrostatic weighing (one-third size).

by wear, if they are taken up in the ordinary way with a pzir of tongs. This risk is obviated by their being kept in the pans when lifted. Two light pans are used of as nearly as possible equal weight, each of which has a loop of wire forming an arch with the ends attached to the opposite sides of the pan, so that it can be easily lifted with the curved end of a brass rod. The pans are marked $X$ and $Y$ respectively. By interchanging the weights in the pans after a series of comparisons, and making a second series and taking the mean result, it gives the difference between the two weights, unaffected by any possible difference in the weight of the two pans. This contrivance is especially useful, when either of the weights to be compared consists of several separate weights. It was used by Prof. Miller for all his more important weighings during the construction of the imperial standard pound. 
The advantage possessed by Gauss's method of alternation over Borda's method of substitution has been proved by Prof. Miller as follows :-

Let $\mathrm{P}$ and $\mathrm{Q}$ be two standard weights of the same denomination to be compared, and $\mathrm{C}$ the counterpoise of each.

For Borda's method, let the readings of the index be denoted by $(C, P)$, when $C$ is in the left pan and $P$ in the right pan, and by $(C, Q)$, when $C$ is in the left pan, and $Q$ in the right pan.

For Gauss's method, let $(Q, P)$ denote the readings when $Q$ is in the left pan and $P$ in the right, and $(P, Q)$, when $P$ is in the left pan and $Q$ in the right pan.

Let $e$ be the probable difference between the recorded and the true position of equilibrium, that is to say, the probable error of a single weighing (not of a comparison, which requires two weighings).

Then by Borda's method, $(\mathrm{C}, \mathrm{P})$ has a probable error $e$, and $(C, Q)$ has a probable error $e$; and the two weighings give the value of $P-Q$ with a probable error of $\sqrt{ }\left(e^{2}+e^{2}\right)=e \sqrt{ } 2$.

By Gauss's method, $(Q, P)$ has a probable error $e$, and $(\mathrm{P}, \mathrm{Q})$ has a probable error $e$; and the two weighings give the value of $P-Q$ with a probable error of $\frac{e}{2} \sqrt{2}$.

Thus the probable error of the result of two weighings by Borda's method is twice as great as by Gauss's method.

To obtain a value of $\mathrm{P}-\mathrm{Q}$ by Borda's method with a probable error of $\frac{e}{2} \sqrt{2}$, we must make four comparisons of two wejghings each. Therefore one comparison by the method of Gauss gives as good a result as four comparisons by Borda's method.

The result of this weighing of two standard weights against each other gives only their apparent difference when weighed in air. In order to ascertain their true difference, it becomes necessary to determine the weight of air displaced by each, from the data which have bcen already mentioned, and to allow for any difference of weight of air displaced, according to the following formula :-

If the weights $P$ and $Q$ appear to be equal in air, the weight of $\mathrm{P}$ - weight of air displaced by $\mathrm{P}$ is equal to the weight of $Q$ - weight of air displaced by $Q$.

In determining the weight of ordinary atmospheric air in rooms where standard weights are compared, and con taining a certain quantity of aqueous vapour and carbonic acid, the practice has been to take, as the unit of weight of air, a litre of dry atmospheric air free from carbonic acid, $=\mathrm{I}^{\circ} 2932227$ gramme, at $0^{\circ} \mathrm{C}$., as determined by Ritter from the observations of $M$. Regnault in Paris, lat. $48^{\circ} 50^{\prime} 14^{\prime \prime}$, and 60 metres above the level of the sea, under the barometric pressure of 760 millimetres of mercury. Assuming that atmospheric air contains, on an average, carbonic acid equal to 0.0004 of its volume, and the density of carbonic acid gas being 1.529 of that of atmospheric air, the weight of a litre of dry atmospheric air containing its average amount of carbonic acid, under the stated circumstances, is $I^{\prime} 2934963$ gramme.

Allowance should be made for the difference of the force of gravity in latitudes other than Paris, as well as for the difference of height of the place of observation above the mean level of the sea. Although the absolute weight varies with the latitude and with the height above or below the mean level of the sea, yet this variation is not felt in the comparison of standard weights in a vacuum, because the weights are equally affected on both sides of the beam. But in all weighings of standards in air re. quiring special accuracy, such variation must be taken into account in computing the weight of air displaced by each standard weight.

Mr. Baily has shown from his pendulum experiments * that if we take $G$ to denote the force of gravity at the mean level of the sea in lat. $45^{\circ}$, the force of gravity in lat. $\lambda$, at the mean level of the sea

$$
=\mathrm{G}(\mathrm{r}-0.0025659 \cos 2 \lambda) \text {. }
$$

And Poisson $t$ has proved that the force of gravity in a given latitude at a place on the surface of the earth at the height $z$ above the mean level of the sea-

$=\left\{1-\left(2-\frac{3}{2} \rho^{\prime}\right) \frac{z}{r}\right\} \times \begin{aligned} & \text { (force of gravity at the mean } \\ & \text { level of the sea in the same lat.) }\end{aligned}$

where $r$ is the radius of the earth, $\rho$ its mean density, and $\rho^{\prime}$ the density of that part of the earth which is above the mean level of the sea. If as is probable,-

$\rho^{\prime}: \rho=5: x \mathrm{I} ; 2-\frac{3}{2} \rho^{\prime}=\mathrm{I} \cdot 32$ nearly $r=6366 \mathrm{I} 98$ metres,

it follows that the weight in grammes of a litre of dry atmospheric air containing the average amount of carbonic acid, at $0^{\circ}$, and under the pressure of 760 millimetres of mercury at $0^{\circ}$, at the height $z$ above the mean level of the sea in lat. $\lambda$ is-

$$
\text { I.2930693 (I - I'32 } \left.\frac{z}{r}\right)(1-0.0025659 \cos 2 \lambda) \text {. }
$$

At Cambridge, where Prof. Miller's observations for determining the weight of the new standard pound were made, in lat. $52^{\circ} 12^{\prime} 18^{\prime \prime}$, about 8 metres above the mean level of the sea (and for which place his tables were computed,) the weight of a litre of dry air containing the average quantity of carbonic acid was found by him to be I'293893 gramme. This weight of air is therefore a little greater than at Paris. From similar data, after taking a further correction by Lasch of the weight of a litre of dry air at Paris $=1 \cdot 293204$ gramme, the weight of a litre of dry air at Berlin (lat. $52^{\circ} 30^{\prime}$, and 40 metres above mean sea level) has been computed to be r'29388 gramme.

The co-efficient of expansion of air under constant pressure between $0^{\circ}$ and $50^{\circ} \mathrm{C}$. is taken from Regnauit's determination to be 0.003656 for $\mathrm{I}^{\circ} \mathrm{C}$., in other words between $0^{\circ}$ and $50^{\circ} \mathrm{C}$, the ratio of the density of air at $0^{\circ}$ to its density at $t^{\circ}$ is I $+0.003656 t$.

With regard to the barometric pressure of the air and the allowance to be made for the pressure of vapour present in it, the density of the vapour of water is determined to be 0.622 of that of air ; that is to say, the ratio of the density of the vapour of water to that of air is I - 0.378 .

Hence, if $t$ be the temperature of the air, $b$ the barometric pressure, 2 the pressure of the vapour present in the air, $b$ and $v$ being expressed in millimetres of mercury at $0^{\circ} \mathrm{C}$, the weight of a litre of air at Cambridge becomes

$$
\frac{1 \cdot 293893}{i+0.003656 t} \frac{b-0.378 v}{760}
$$

The ratio of the density of air to the maximum density of water is found by dividing the above expression by $I, 000$, as a litre of water is the volume of 1,000 grammes of water at its maximum density. Prof. Miller's Table I. gives the logarithms of this ratio at the normal barometric pressure of 760 millimetres, at the several degrees of temperature from $0^{\circ}$ to $30^{\circ}$. These logarithms require to be diminished only by 0.000026 for weighings at the Standards Office, Westminster, lat. $51^{\circ} 30^{\prime}$, and about 5 metres above the mean sea-level; and when dimi-

* "Memoir of the Astronomical Society," vol. vii. p. 94 . † "Memoires de l'Institut," tome xxi. pp. 91, 238 . 
nished by 0.000132 , they may be used for the reductions of weighings at Paris.

The values of the pressure of vapour at the same temperatures in millimetres of mercury at $0^{\circ}$, according to Regnault's observations, are stated by Prof. Miller in a separate Table II. These values are given on the assumption that the pressure of vapour in rooms that are not heated artificially is two-thirds of the maximum pressure of vapour due to the temperature, as shown by the results of experiments on the authority of Biot, Regnault, and Bianchi.

The actual mode of ascertaining the weight of air displaced by two standard weights may now be described.

For determining the temperature of the air and of the two standard weights during the weighings, two standard thermometers are placed in the balance case, and their readings noted at the beginning and end of the weighings. The weight of air displaced by each of two standard weights is to be ascertained by the following formula :

Log. weight in grains of air displaced by $\mathrm{P}=\log . / 2+$ $\log$. A $t+\log .(\mathrm{I}+e \mathrm{P} t)+\log$. weight of $\mathrm{P}$ in grains $\log \triangle \mathrm{P}$.

Here $t$ denotes the temperature of the air by the Centigrade thermometer;

$b$ the barometric pressure of the air in millimetres of mercury at $0^{\circ} \mathrm{C}$. ;

o the maximum pressure of aqueous vapour contained in the air, also in millimetres of mercury;

$h=b-0.378 \times \frac{2}{5} v$;

At the ratio of density of air at to to the maximum density of water ;

$c \mathrm{P} t$ the allowance for expansion in volume of $\mathrm{P}$, or the ratio of its density at $0^{\circ}$ to its density at $t$;

$\triangle P$ the ratio of density of $P$ at $0^{\circ}$ to the maximum density of water.

By this formula, the required result is to be obtained. The logarithms of the three first terms may be found in Prof. Miller's tables, pp. 785-79r of his account of the construction of the new standard pound, Phil. Trans., part iii. of 1856 .

Reference has already been made to the mode of ascertaining the volume or density of a standard weight by determining the difference of its weight in air and in water. The following practice for all such hydrostatic weighings was adopted by Prof. Miller when determining the densities of all the standard weights constructed under the sanction of the Commission for restoring the Imperial Standards, and is also followed in the Standards Department. In this process it is requisite to employ pure distilled water, and with this object the water used in the Standards Department is twice distilled in a still of the best construction, erected in the office, and the best chemical tests are employed for ascertaining that the water is free from any foreign substances.

The vessel for containing the distilled water is a glass jar, rather more than 6 inches in internal height and diameter. A stout copper wire is stretched across the mouth of the jar (see Fig. I8) in such a manner as to leave a circular space in the middle, large enough to admit the passage of the standard weight $P$, the density of which is to be ascertained. This copper wire supports two thermometers, adjustable as to their height, for determining the temperature of the water at the mean height of $\mathrm{B}$ during the weighings. It also serves to sustain a glass tube, open at both ends, and placed close to the side of the jar. A small glass funnel is inserted in the upper part of the tube, and in the lower part are one or two pieces of clean sponge.

The standard weight $\mathrm{P}$ is suspended from a hook under the right pan of the balance, specially constructed for hydrostatic weighings. A fine copper wire, the weight of which per inch is known, is attached to the hook by a loop, and has another loop at the other end, To this lower loop is attached a stout wire, bent and terminating in a double hook, which fits round $P$, and holds it securely. The counterpoise of $P$ is next placed in the left pan of the balance. The glass jar is placed under the right pan of the balance, $\mathrm{P}$ being suspended in it, and the water is gently poured into the funnel and the jar filled to the requisite height above $P$. The bubbles of air are arrested by the pieces of sponge, and, ascending up the glass tube, are thus prevented from entering the jar. It is of importance to ascertain that no bubble of air is attached to $P$, and if so, it may generally be removed by the feather of a quill. But it sometimes happens that the weight $P$ has an irregular surface, and air attaching to it cannot be thus dislodged. In such cases a small bell-shaped glass jar just large enough to hold $\mathrm{P}$ and its supporting wire, is used. This vessel is filled with water sufficient to cover $P$, and is suspended over the flame of a spirit lamp by a stout wire, bent at its lower end into a ring, into which the jar clescends to its rim, and the water is allowed to boil until it is seen that the air has been entirely expelled. When cooled, the small jar containing $P$ is immersed iu the water, which nearly fills the large jar, and the small jar, with its wire, is then disengaged and lowered till $P$ hangs clear of it, when it is removed. The transfer of $P$ from the small to the large jar is thus effected without taking it out of the water.

For the actual weighing of $\mathrm{P}$ in water, after it has been counterpoised in air, weights equal to the difference of weight of $P$ in water and in air, are placed in the right pan till equilibrium is produced, when the readings of the scale are observed. P is next removed, leaving its hook suspended in the water, and a volume of water equal to the volume of $P$ is added to the water in the jar, so as to leave the same quantity of wire immersed as before. The requisite weights are then added to the right pan, until the equilibrium, which has been disturbed by the removal of $\mathrm{P}$, is again produced, when the reading of the scale is observed and noted. This gives the actual weight in water of $P$.

The thermometers in the water are so placed as to give the temperature of the water at the centre of gravity of $P$. Another thermometer is placed in the balance case to give the temperature of the air during the weighings. The reading of the barometer is also noted.

Having determined the weight of $\mathrm{P}$ in air of ascertained density, its volume and ciensity are calculated according to the following formula, the unit of volume being the volume of a grain weight of water at its maximum density :--

Let $P$ in water at $t^{\circ}$ appear to weigh as much as $Q$ in air: Then the weight of water at $t^{\circ}$ displaced by $\widetilde{\mathrm{P}}=$ weight of $P$ - weight of $Q+$ weight of air displaced by Q.

Log. volume of $\mathrm{P}=$ weight in grains of the water displaced by $\mathrm{P}+\log$. $\mathrm{W}_{\mathrm{t}}-\log$. $\left(\mathrm{I}+e \mathrm{P}_{\mathrm{t}}\right)$; where $\mathrm{W}_{\mathrm{t}}$ is the ratio of the maximum density of water to its density at $t$, and $e \mathrm{P} t$ is the expansion in volume of $\mathrm{P}$ at $t$. (The logarithms of these values are given in tables.)

$\mathrm{Log}$. density of $P=\log$. weight of $P$ in grains $-\log$. volume of $\mathrm{P}$.

The actual weight of air displaced is to be ascertained by the method already stated.

As the true weight of $P$ in air cannot be ascertained until its volume or density is known, an approximate value of the volume of $\mathrm{P}$ may be found by assuming the weight of $\mathrm{P}$ to be equal to its apparent weight in air; and this value of the volume of $P$ may be used in reducing the weight of $P$, and thus a more accurate value of the volume of $\mathrm{P}$ obtained, by means of which a closer approximation to the values of the absolute weight of $P$, and of its density may be found. This process should be repeated when greater exactness is required.

H. W. CHISholait 